\title{
Measles Outbreak among Previously Immunized Adult Healthcare Workers, China, 2015
}

\author{
Zhengyi Zhang, ${ }^{1}$ Yuan Zhao, ${ }^{1}$ Lili Yang, ${ }^{1}$ Changhong Lu, ${ }^{1}$ Ying Meng, ${ }^{1}$ Xiaoli Guan, \\ Hongjin An, ${ }^{1}$ Meizhong Zhang, ${ }^{2}$ Wenqin Guo,, Bo Shang, ${ }^{1}$ and Jing Yu ${ }^{3}$ \\ ${ }^{1}$ Department of General Medicine, Lanzhou University Second Hospital, Gansu 730030, China \\ ${ }^{2}$ Department of Public Health, Lanzhou University Second Hospital, Gansu 730030, China \\ ${ }^{3}$ Department of Cardiology, Lanzhou University Second Hospital, Gansu 730030, China \\ Correspondence should be addressed to Jing Yu; yujing2304@126.com
}

Received 22 January 2016; Revised 15 March 2016; Accepted 27 March 2016

Academic Editor: Mark A. Wainberg

Copyright (C) 2016 Zhengyi Zhang et al. This is an open access article distributed under the Creative Commons Attribution License, which permits unrestricted use, distribution, and reproduction in any medium, provided the original work is properly cited.

\begin{abstract}
Measles is caused by measles virus belonging to genus Morbillivirus of the family Paramyxoviridae. Vaccination has played a critical role in controlling measles infection worldwide. However, in the recent years, outbreaks of measles infection still occur in many developing countries. Here, we report an outbreak of measles among healthcare workers and among the 60 measles infected patients 50 were healthcare workers including doctors, nurses, staff, and medics. Fifty-one patients (85\%) tested positive for IgM antibodies against the measles virus and 50 patients $(83.3 \%)$ tested positive for measles virus RNA. Surprisingly, $73.3 \%$ of the infected individuals had been previously immunized against measles. Since there is no infection division in our hospital, the fever clinics are located in the Emergency Division. In addition, the fever and rash were not recognized as measles symptoms at the beginning of the outbreak. These factors result in delay in isolation and early confirmation of the suspected patients and eventually a measles outbreak in the hospital. Our report highlights the importance of following a two-dose measles vaccine program in people including the healthcare workers. In addition, vigilant attention should be paid to medical staff with clinical fever and rash symptoms to avoid a possible nosocomial transmission of measles infection.
\end{abstract}

\section{Introduction}

Measles is a highly infectious respiratory disease that occurs worldwide, which mainly causes morbidity and mortality among children. Its causative agent, the measles virus, is a single stranded negative sense RNA virus belonging to the Paramyxoviridae family [1]. The clinical symptoms of measles include high fever, a maculopapular rash, conjunctivitis, cough, and coryza; furthermore, its complications can include pneumonia, blindness, and brain damage [2]. The incubation period of the disease ranges from 6 to 21 days, and its transmission modes include airborne droplet transmission and direct contact with the respiratory secretions of an infected individual or their fomites [2]. An infected individual can disseminate the virus to others 2 days before and 5 days after the onset of their own clinical symptoms. Vaccine immunization has played an important role in preventing, controlling, and eradicating infectious diseases such as poliomyelitis. Since the 1960 s, a single dose of liquid measles vaccine has been administered to infants aged $>8$ months in China [3]. Starting in 1978, a national Expanded Program on Immunization (EPI) was initiated to ensure that all infants received one dose of measles vaccine at the age of 8 months. In 1985, an amended two-dose vaccination schedule was established, in which the first dose of vaccine is administered at the age of 8 months and the second dose at the age of 7 years [4]. In 2005, China took action to eliminate measles in the Western Pacific Region by 2012 (a goal set by the World Health Organization) and changed the age for administration of the second vaccine dose from 7 years to 18 months [5]. Use of the measles vaccine has resulted in substantial reductions in the incidence and mortality of measles worldwide, including China [6]. In addition to the EPI, a nationwide measles Supplementary 
Immunization Activity (SIA) was initiated in 2010 to close immunization gaps among children and in other populations [3]. In 2012, the SIA program had helped to reduce the incidence of measles to its lowest recorded level (6183 cases, $4.6 \mathrm{cases} / \mathrm{million}$ total population) [7]. However, in recent years, there have been several reports of measles infections in adults, raising concerns about the current immunization programs $[8,9]$. Here, we report for the first time a measles outbreak among healthcare workers in mainland China.

\section{Case Presentation}

On March 16, 2015, a patient admitted to the Emergency Department with high fever was diagnosed as having a measles infection. On the day of March 20, 2016, three nurses from Emergency Department who had direct and (or) indirect contact with the patient displayed similar clinical signs including fever and rash that were determined to be positive for measles infection. The hospital noticed that it might be a break of measles infection with possibility of nosocomial transmission, and the first case on March 16,2015 , was suspected to be the source of this outbreak. Immediately, a series of measures for the prevention and control of nosocomial measles according to Guidelines of the People's Republic of China on the Prevention and Control of Infectious Diseases have been set up as follows.

Measures for the Prevention and Control of Nosocomial Measles. Consider the following:

(1) Setting up isolation wards in a well-ventilated old building.

(2) Enhancing ventilation, isolation, and disinfection measures in isolation areas.

(3) Isolating those patients with great transmission potentials including severe-ill patients and students.

(4) Providing measles immunization to a total of 2,400 hospital staff.

(5) Close monitoring of the measles infected patients, paying much more attention to patients with severe complications, and canceling multidisciplinary team meetings and treatment.

First, the isolation ward was set up with a well-ventilated system to prevent possible transmission among community people and the first patient (case number 1) was hospitalized two weeks before discharge. Second, Emergency Department is the unit with serious and multiple illness in which the healthcare staff from other departments who went to consultation directly or indirectly were exposed to the Emergency Department patients with measles; they were infected with measles and/or spread it to other staff in their departments. Therefore, more medical staffs from other departments with similar symptoms and close contact with the known measles cases were searched. Third, reimmunization probably played important roles in containing measles transmission and a total of 2400 hospital staff were given a measles immunization before March 27, 2015. Since then, new measles case numbers

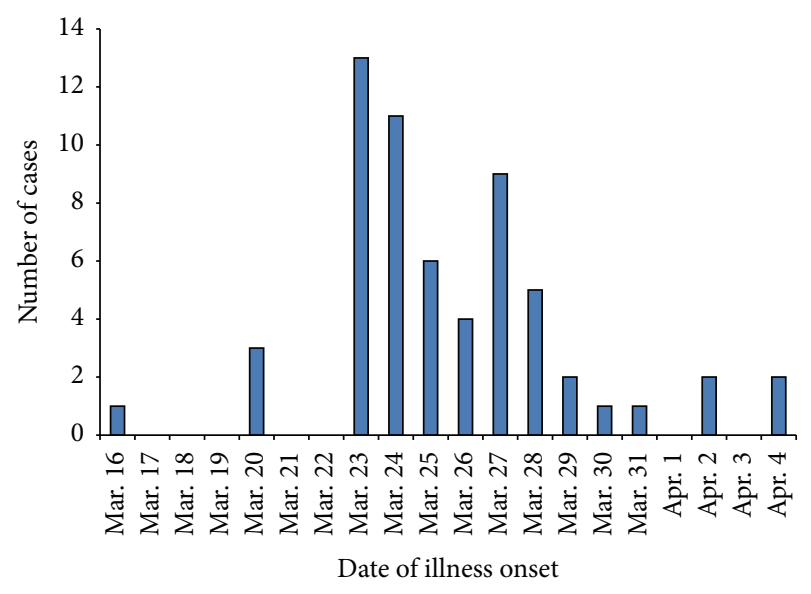

FIGURE 1: An epidemic curve shows the number of cases during the measles outbreak in Lanzhou University Second Hospital between March 16, 2016, and April 4, 2015. The $x$-axis represents date of illness onset; the $y$-axis represents the number of cases.

began to decrease and the last two cases were reported on April 4, 2016 (Figure 1). Among 20 patients admitted in isolation wards, only 6 patients were treated. Due to the mild symptoms in most hospital staff patients, they chose to stay home and no new case was reported among those persons contacted with patients who stayed home. The study was approved by Institutional Review Board (IRB) of the Lanzhou University Second Hospital and written patient consent was obtained under Review Board approval number 2015A-077. Verbal consent was obtained from all participants using an information sheet approved by the IRB of the Lanzhou University Second Hospital.

Patients with clinical symptoms including fever, rash, and/or conjunctivitis were defined as suspected measles cases. Between March 16 and April 4, 2015, both serum and throat swab samples were gathered from 102 suspected measles cases and then tested for measles virus IgM using IgM ELISA test kit (Zhuhai SEZ Haitai Biological Pharmaceuticals Co., Ltd., Zhuhai, China) and for measles virus RNA using a real-time reverse transcription PCR kit (Jiangsu BioPerfectus Technologies Co., Ltd., Jiangsu, China). Finally, 60 suspected measles cases were confirmed as the diagnosis of measles infection among whom there were $51(85.00 \%)$ cases positive for measles IgM, 50 (83.33\%) for measles virus RNA, and $41(68.33 \%)$ for both measles IgM and virus RNA. Among the 60 positive cases, $39(65.00 \%)$ and $21(35.00 \%)$ were in female and male patients, respectively. Seven cases (11.66\%) were aged $1-18$ years, 50 cases $(83.33 \%)$ were aged $21-40$ years, and 3 cases $(5.01 \%)$ were aged $>40$ years (Table 1 ). There were $31(51.66 \%)$ nurses, $11(18.33 \%)$ hospital staff members, 7 (11.66\%) doctors, 1 (1.69\%) medic, and the remaining $10(16.66 \%)$ hospital patients (Table 1). Thus $83.33 \%$ of measles cases were healthcare workers. The majority of cases displayed one or more of the following symptoms: Koplik spots in 36 patients $(60 \%)$, catarrh in 51 patients $(85 \%)$, and conjunctivitis in 50 patients (83.3\%). Only a few patients had severe symptoms, which included pneumonia in 2 patients 
TABLE 1: Summary of cases diagnosed with measles in the outbreak.

\begin{tabular}{|c|c|}
\hline \multicolumn{2}{|l|}{ Gender } \\
\hline Female $(n, \%)$ & $39(65.00 \%)$ \\
\hline Male $(n, \%)$ & $21(35.00 \%)$ \\
\hline \multicolumn{2}{|l|}{ Age } \\
\hline $1-18$ years $(n, \%)$ & $7(11.66 \%)$ \\
\hline $19-40$ years $(n, \%)$ & $50(83.33 \%)$ \\
\hline$>40$ years $(n, \%)$ & $3(5.01 \%)$ \\
\hline \multicolumn{2}{|l|}{ Prevalent location of the measles } \\
\hline New building of the hospital $(n, \%)$ & $60(100 \%)$ \\
\hline Old building of the hospital $(n, \%)$ & $0(0.00 \%)$ \\
\hline \multicolumn{2}{|l|}{ Occupation } \\
\hline Nurse $(n, \%)$ & $31(51.66 \%)$ \\
\hline Staff $(n, \%)$ & $11(18.33 \%)$ \\
\hline Clinical patient $(n, \%)$ & $10(16.66 \%)$ \\
\hline Doctor $(n, \%)$ & $7(11.66 \%)$ \\
\hline Medic $(n, \%)$ & $1(1.69 \%)$ \\
\hline \multicolumn{2}{|l|}{ Vaccination history } \\
\hline Vaccination $(n, \%)$ & $44(73.33 \%)$ \\
\hline Unknown $(n, \%)$ & $12(20.00 \%)$ \\
\hline No vaccination $(n, \%)$ & $4(6.67 \%)$ \\
\hline \multicolumn{2}{|l|}{ Positive rate of the test method } \\
\hline $\operatorname{MV} \operatorname{IgM}(n, \%)$ & $51(85.00 \%)$ \\
\hline $\operatorname{MV} \operatorname{PCR}(n, \%)$ & $50(83.33 \%)$ \\
\hline Both $(n, \%)$ & $41(68.33 \%)$ \\
\hline \multicolumn{2}{|l|}{ Clinical manifestation } \\
\hline Koplik spots $(n, \%)$ & $36(60.00 \%)$ \\
\hline Catarrh $(n, \%)$ & $51(85.00 \%)$ \\
\hline Conjunctivitis $(n, \%)$ & $50(83.33 \%)$ \\
\hline \multicolumn{2}{|l|}{ Complication $(n, \%)$} \\
\hline Pneumonia $(n, \%)$ & $2(3.33 \%)$ \\
\hline Laryngitis $(n, \%)$ & $1(1.67 \%)$ \\
\hline Liver dysfunction $(n, \%)$ & $6(10.00 \%)$ \\
\hline
\end{tabular}

(3.3\%) and liver dysfunction in 6 patients (10\%) (Tables 1 and 2).

\section{Discussion}

The results of a recent study of measles infections in China indicated that measles usually occurred in young unvaccinated children [7]; however, the measles outbreak in our hospital mainly involved adults aged between 20 and 40 years. Surprisingly, 44 of the 60 infected patients $(73.33 \%)$ had received a single dose measles vaccination during their childhood, and only one patient (1.67\%) received a second immunization at the age of 18 years. Thus while $>70 \%$ of the patients had been previously immunized against measles, they remained susceptible to infection with the measles virus. This finding suggests that the level of protection provided by a single measles immunization may drop to baseline and cease to protect against the disease. The possibility of onedose primary vaccine failure in the patients that led to their susceptibility to measles can not be excluded. Moreover, two previous studies reported that measles outbreaks in Beijing and Hangzhou mainly occurred among people aged $>15$ years. However, $87.6 \%$ and $66.7 \%$ of the adults infected in those two outbreaks, respectively, had unknown vaccination histories [8, 9]. More importantly, several measures should be immediately taken to prevent and control measles infection and transmission in hospitals. These measures include increasing the room ventilation in all departments, promptly isolating suspected cases from other patients, establishing a program for evaluating the measles immune status of all hospital healthcare workers, and providing a second vaccination when appropriate and all measles-susceptible individuals should be vaccinated immediately following contact with a suspected case of measles [10].

\section{Conclusion}

In summary, our study reports a measles outbreak that occurred among adult healthcare workers who were previously underimmunized against measles. It highlights that healthcare workers need to be fully vaccinated through a 2dose measles vaccine program, which could greatly prevent nosocomial outbreak of measles. Equally important, fever clinics should be separated from other divisions in the hospital. Vigilant attention should be paid to the patients with the clinical fever and rash symptoms and early isolation and definite diagnosis of the measles suspected medical staff patients to avoid a possible nosocomial transmission of measles infection.

\section{Disclosure}

Dr. Zhang is the director of the Department of General Medicine, Lanzhou University Second Hospital, Gansu, China. His research interest is cardiovascular disease.

\section{Competing Interests}

The authors declare that there are no competing interests regarding the publication of this paper.

\section{Authors' Contributions}

All authors read and approved the final paper. Zhengyi Zhang, Yuan Zhao, and Jing Yu conceived and designed the experiments. Zhengyi Zhang, Yuan Zhao, Lili Yang, Changhong Lu, Ying Meng, Xiaoli Guan, and Hongjin An performed the experiments. Zhengyi Zhang, Yuan Zhao, Meizhong Zhang, Wenqin Guo, and Jing Yu analyzed the data. Zhengyi Zhang, Yuan Zhao, and Jing Yu wrote the paper. Zhengyi Zhang and Yuan Zhao contributed equally to this work. 


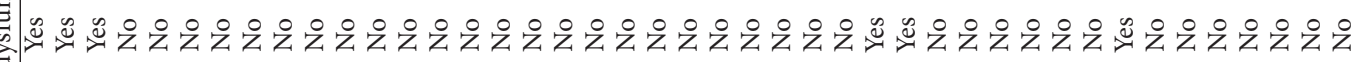

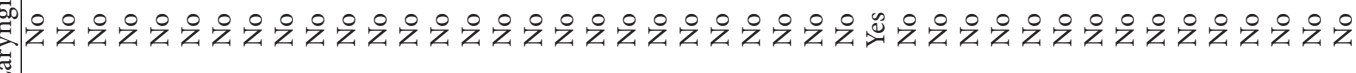

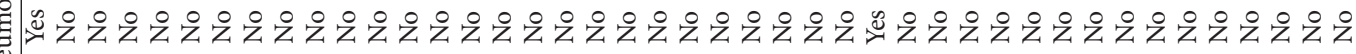

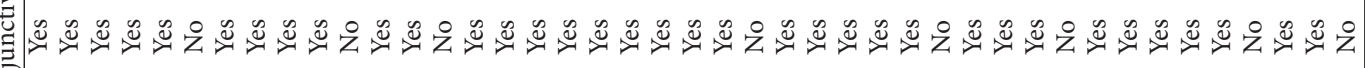

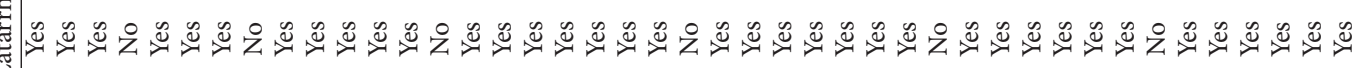

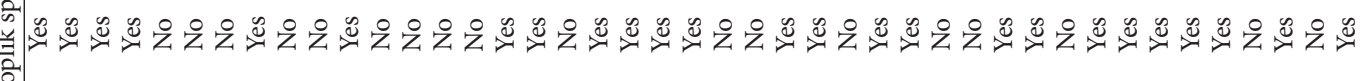

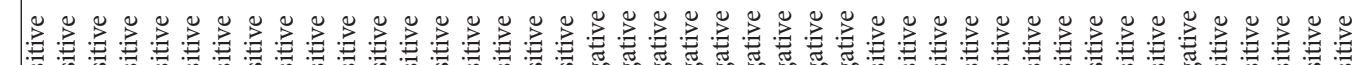

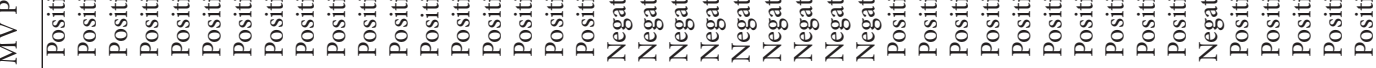

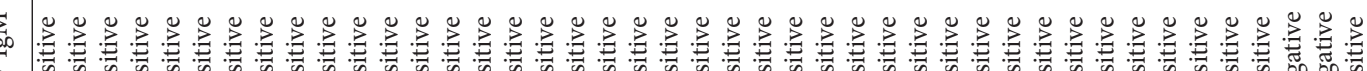

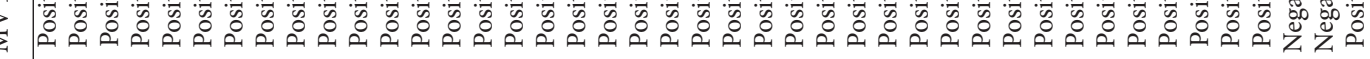

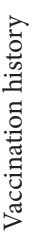

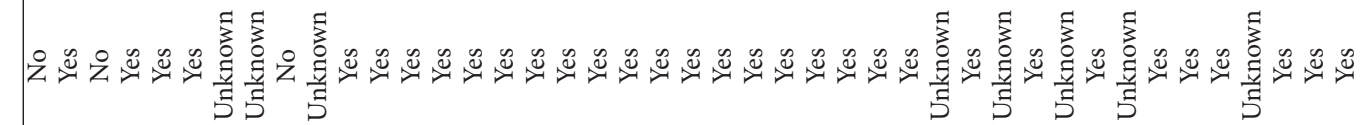

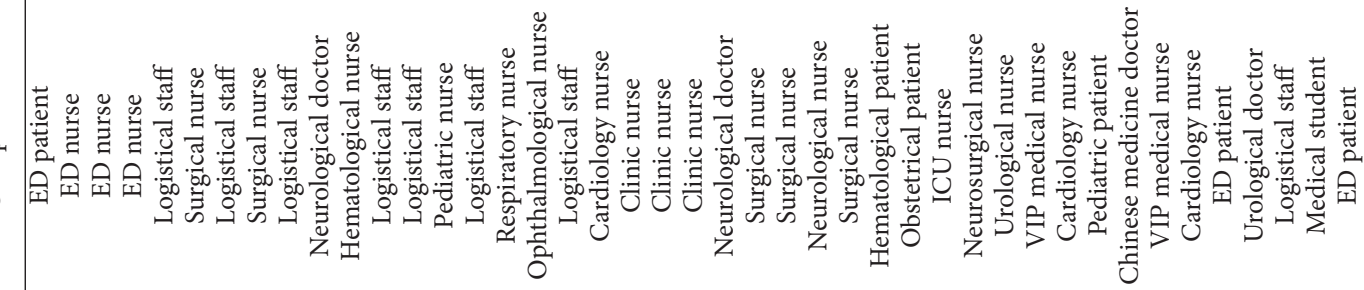
离

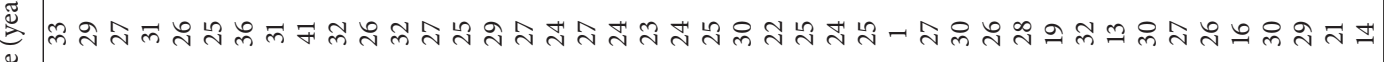
总

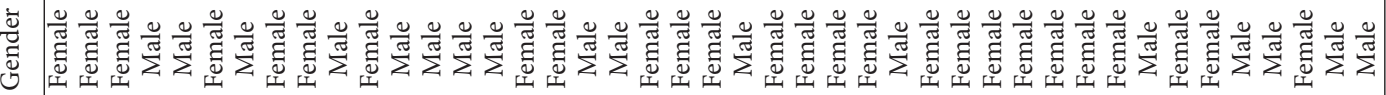

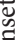

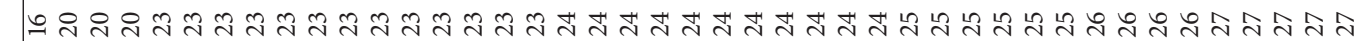

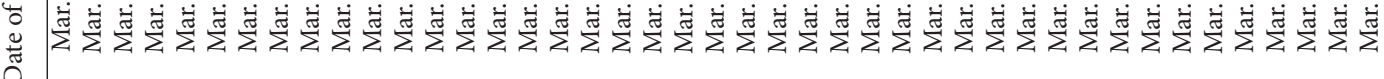
峁 空 - 


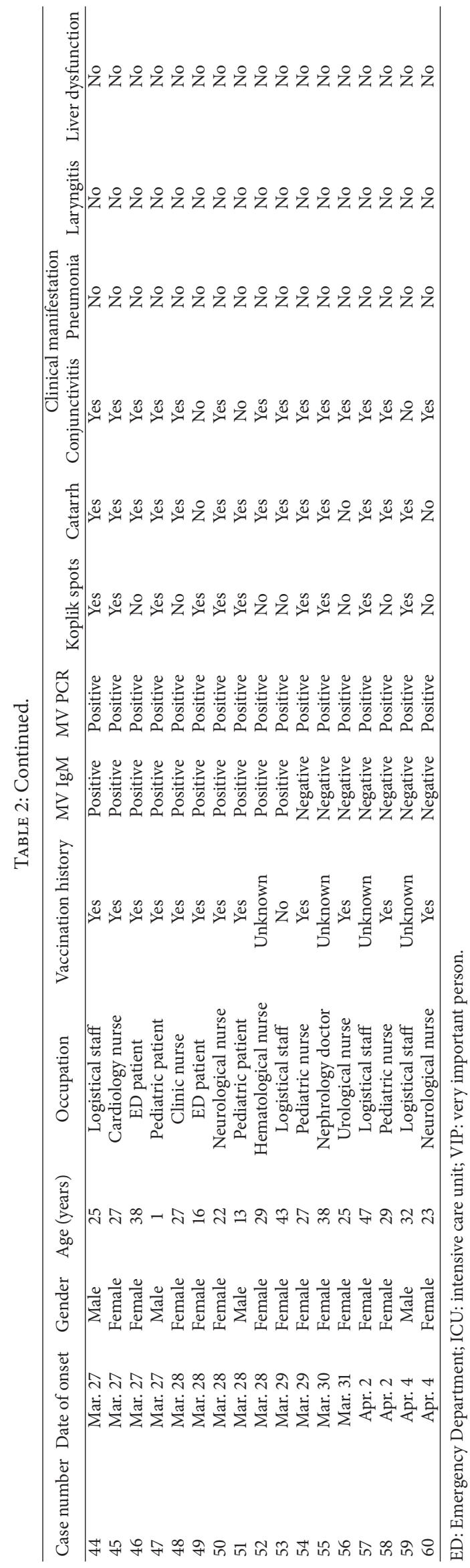




\section{Acknowledgments}

The authors would like to thank staff in Department of Public Health and Department of Medical Affairs for providing data on measles outbreak. Thanks extended to Lanzhou Center for Disease Control and Prevention for technical help on the measles diagnostic assays.

\section{References}

[1] T. Duke and C. S. Mgone, "Measles: not just another viral exanthem," The Lancet, vol. 361, no. 9359, pp. 763-773, 2003.

[2] R. T. Perry and N. A. Halsey, "The clinical significance of measles: a review," Journal of Infectious Diseases, vol. 189, supplement 1, pp. S4-S16, 2004.

[3] C. Ma, Z. An, L. Hao et al., "Progress toward measles elimination in the People's Republic of China, 2000-2009," Journal of Infectious Diseases, vol. 204, no. 1, pp. S447-S454, 2011.

[4] J. Gao, E. Chen, Z. Wang et al., "Epidemic of measles following the nationwide mass immunization campaign," BMC Infectious Diseases, vol. 13, article 139, 2013.

[5] C. Ma, F. Li, X. Zheng et al., "Measles vaccine coverage estimates in an outbreak three years after the nation-wide campaign in China: implications for measles elimination, 2013," BMC Infectious Diseases, vol. 15, article 23, 2015.

[6] De la Santé, "Global reductions in measles mortality 2000-2008 and the risk of measles resurgence," Weekly Epidemiological Record, vol. 84, no. 49, pp. 509-516, 2009.

[7] C. Ma, L. Hao, Y. Zhang et al., "Monitoring progress towards the elimination of measles in China: an analysis of measles surveillance data," Bulletin of the World Health Organization, vol. 92, no. 5, pp. 340-347, 2014.

[8] M. Chen, Y. Zhang, F. Huang et al., "Endemic and imported measles virus-associated outbreaks among adults, Beijing, China, 2013," Emerging Infectious Diseases, vol. 21, no. 3, pp. 477479, 2015.

[9] F.-J. Wang, X.-J. Sun, F.-L. Wang, L.-F. Jiang, E.-P. Xu, and J.-F. Guo, "An outbreak of adult measles by nosocomial transmission in a high vaccination coverage community," International Journal of Infectious Diseases, vol. 26, pp. e67-e70, 2014.

[10] E. Botelho-Nevers, P. Gautret, R. Biellik, and P. Brouqui, "Nosocomial transmission of measles: an updated review," Vaccine, vol. 30, no. 27, pp. 3996-4001, 2012. 


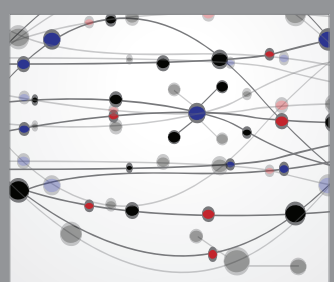

The Scientific World Journal
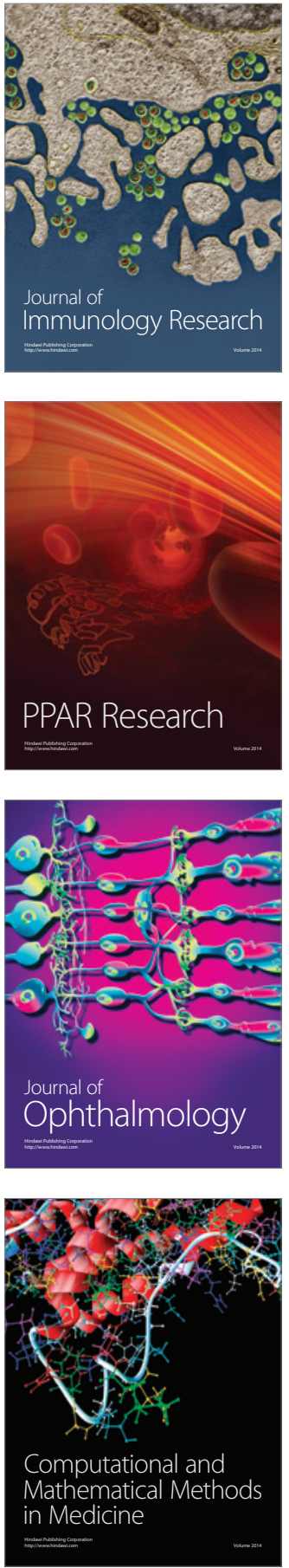

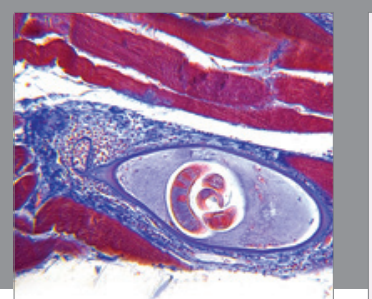

Gastroenterology Research and Practice

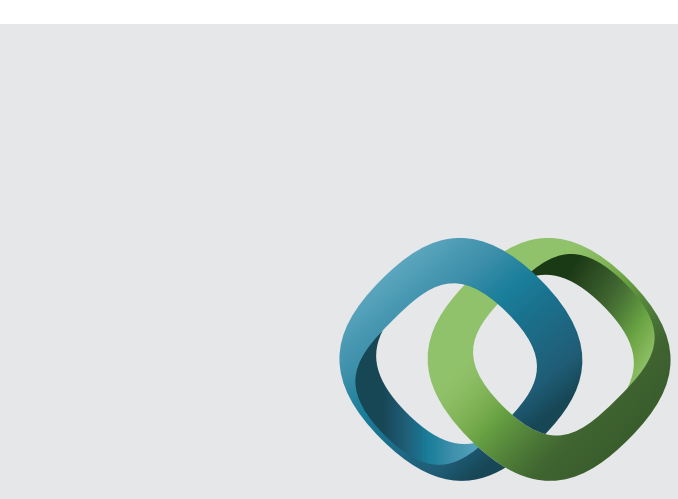

\section{Hindawi}

Submit your manuscripts at

http://www.hindawi.com
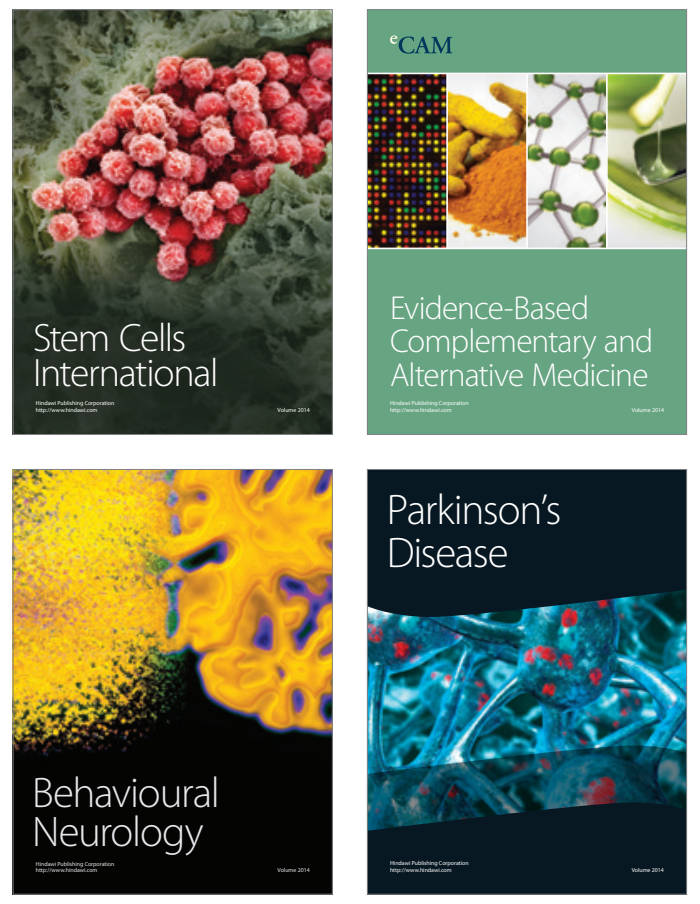
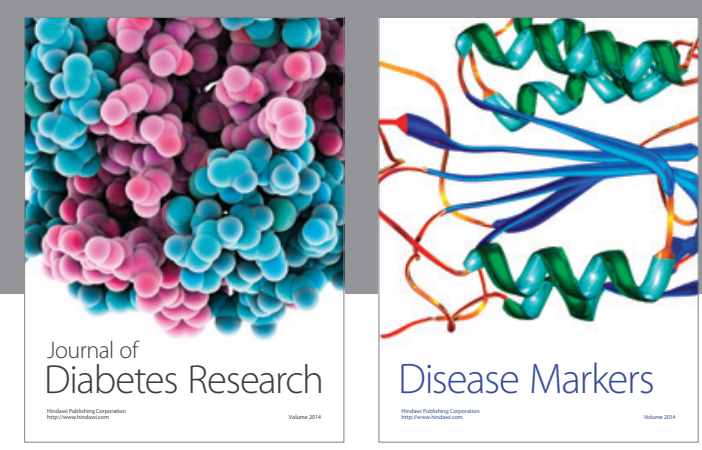

Disease Markers
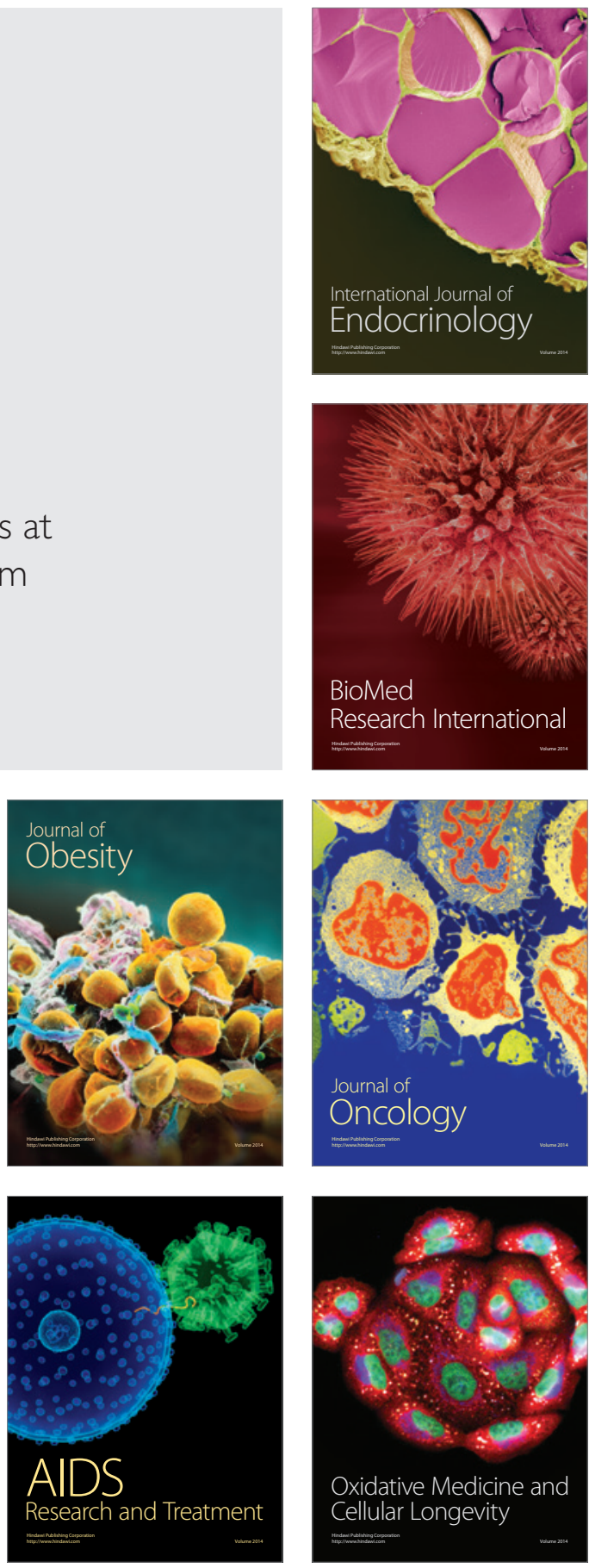